\title{
TRANSPORT OF A SINGLE SPHERICAL PARTICLE IN LOW REYNOLDS NUMBERS' LINEAR SHEAR FLOWS: EXPERIMENT AND MODELING
}

\author{
PETER VOROBIEFF ${ }^{1}$, NIMA FATHI $^{1}$, SEYED SOBHAN ALEYASIN $^{2} \&$ GOODARZ AHMADI $^{3}$ \\ ${ }^{1}$ Department of Mechanical Engineering, University of New Mexico, USA \\ ${ }^{2}$ School of Mechatronic Systems Engineering, Simon Fraser University, Canada \\ ${ }^{3}$ Mechanical and Aeronautical Engineering, Clarkson University, USA
}

\begin{abstract}
Trajectories of a buoyant spherical solid particle in a linear shear flow were investigated at low Reynolds numbers. A two-dimensional CFD analysis was performed to simulate the solid-fluid flow. Our numerical model uses the discrete phase element method (DPM) to simulate the fluid domain and particle (solid phase) motion. The reliability of the computational results was evaluated for the particle trajectory acquired at the University of New Mexico's stratified linear shear flow generator. The agreement between the numerical results with the experimental data is quantified.
\end{abstract}

Keywords: particle migration, multiphase flows, linear shear flows, CFD.

\section{INTRODUCTION}

A fluid-solid flow is an interdisciplinary research area with many technological, commercial and medical applications. Suspensions of macro- to nano-scale particles in viscous fluid flows occur in transport of sediments in rivers and estuaries, to the use of nano-fluids as high-performance coolants. Other important applications are contaminant transport and exposure assessment and slurries flows, as well as secondary oil recovery by hydraulic fracturing. The behavior of solid particles in a viscous fluid is one of the oldest classical problems in this field. One of the earliest investigations of the motion of small particles in a viscous fluid at low Reynolds number (Re) was performed by Stokes in 1851 [1]. More recently, Ingber [2] investigated a variety of particle interactions including particle/particle, wall/particle and particle migration in nonlinear shear fields. A semi-analytical solution was developed for the motion of two spherical particles suspended in an unbounded arbitrary shear flow [3]. Comparisons of computational prediction of single and double particle trajectories using the discrete phase model (DPM) against the semi-analytical solution were performed [4]-[6]. In addition to DPM, several particle methods including smoothed particle hydrodynamics, smoothed profile hydrodynamics and the modified version of the front tracking method have been utilized to investigate the migration of macro- to micro-scale droplets, bubbles and solid particles in fluid flows [7]-[11]. In these numerical approaches, accurate evaluation of the discontinuities between the primary and the secondary phases can be challenging.

The multiphase nature of the flow introduces several problems that are absent in the single-phase formulation. First, from the computational point of view, it greatly adds to the modeling complexity, because flow features must be resolved for a greater range of scales to account for the fluid-particle interaction [2]. The situation when two particles come into contact inside the fluid is especially challenging, because for two idealized spherical particles, a point of contact is a geometric singularity that can produce undesirable mesh artifacts.

The same problem (particles in contact or in near-contact) also has physical implications that may still require elucidation. Particle interaction dominated by non-hydrodynamic forces 
will lead to irreversibility of such interactions even at very low Reynolds numbers, where the hydrodynamics would be nominally reversible (Stokesian). The extent of this irreversibility was shown to be directly related to the particle surface properties on the microscale (e.g., average roughness) [12]. Two interacting particles in a nominally reversible shear flow do not return to their original positions. For three interacting particles, it was shown that their behavior becomes chaotic [13], [14]. Irreversibility in individual particle interactions can also be related to the behaviors of large group of particles, such as changes in the particle distribution profiles in sheared flows [15]. Finally, even a single particle in fluid flow can manifest interesting and sometimes perplexing behaviours, such as thermophoresis [16].

In this article, the new development of our linear shear flow in the Couette flow apparatus is presented. The modified arrangement makes it possible to study the single and multi-particles migration in parallel and counter Couette flows. Compared to the previous facilities, the new device also provides higher accuracy for setting the boundary conditions for making fluid and particle measurements.

\section{EXPERIMENTAL AND COMPUTATIONAL PROCEDURE}

Experiments are conducted to determine the behavior of a single spherical particle suspended in linear shear flow. Here the particle was comprised of polymethyl-metacrylate (PMMA). It was nominally spherical, with a diameter of $6.35 \mathrm{~mm}$, and was suspended in a tank between two gravity-stratified layers of fluids with approximately equal viscosity but with different densities.

Fig. 1 shows the experimental apparatus including the tank and the compumotor which is a computer-controlled stepper motor. The compumotor was used to displace the belts.

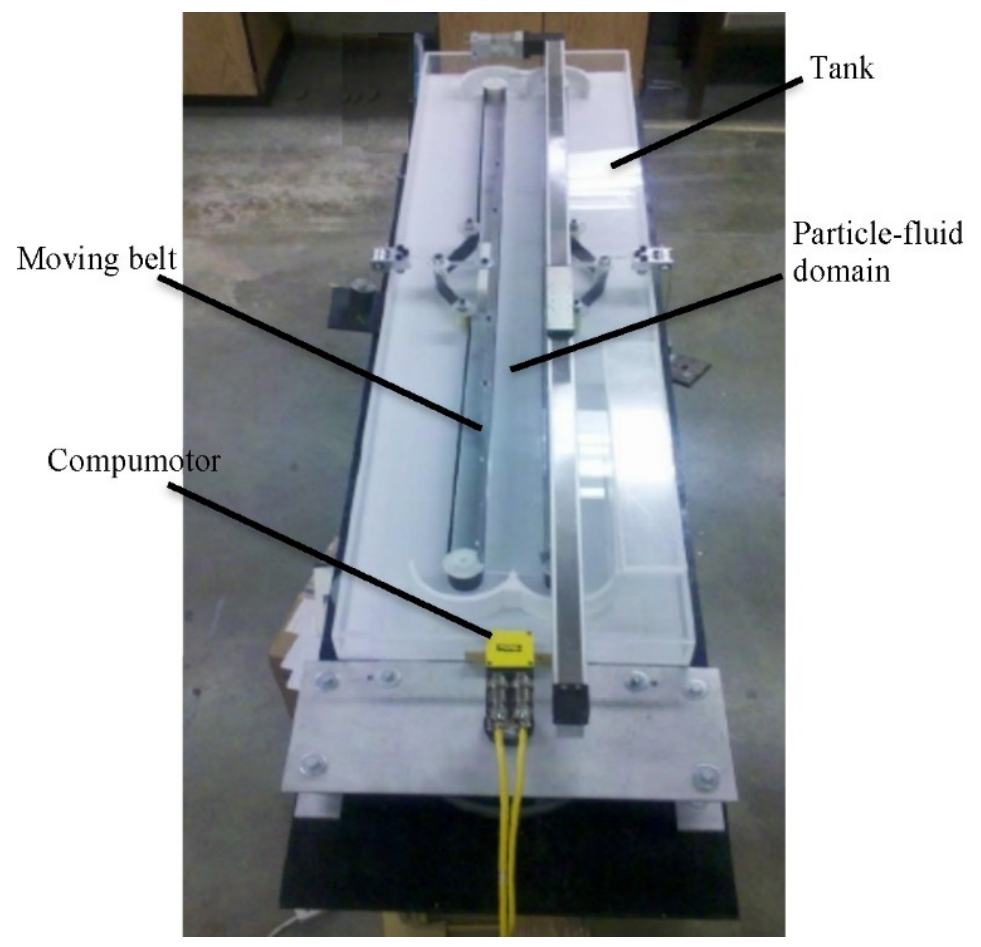

Figure 1: Experimental apparatus. 
The particle was placed between two belts as shown in the top picture in Fig. 2. The schematic representation of the experiment showing the boundary conditions of the physical domain and the suspended particle between the two layers is also shown in Fig. 2. After the particle is settled in the desired position, the side belts are moved at a constant velocity to provide the fluid domain with a shear field in the Couette flow configuration.

As mentioned before, a computer-controlled stepper motor is used to provide the moving boundary conditions via the moving belts. The rectangular tank is filled with a stratified viscous fluid which is a water solution of $\mathrm{ZnCl}_{2}$ and Triton X100. The material properties of the primary phase - upper and lower layer - are evaluated and are shown in Table 1. The same stratified fluid components were also used to study the migration of pairs of nearly spherical solid particles suspended in a shear flow inside a Couette cell for the Reynolds number of approximately 0.1 [12], [17].

To model and simulate the fluid domain including the solid particle phase, the standard discrete phase model is used [18]. The Lagrangian DPM is based on a translational force balance that is formulated for an individual particle. In the DPM, typically the particle is subject to gravity, drag, pressure, Magnus, virtual mass and Saffman forces.

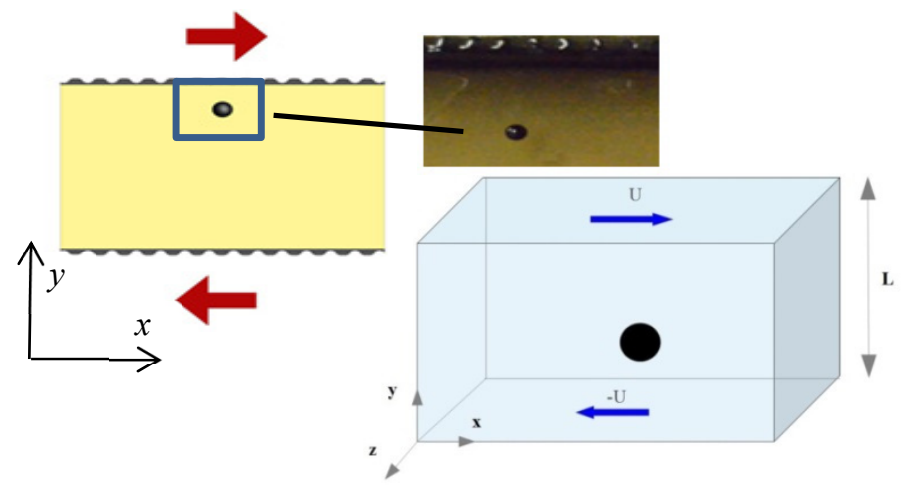

Figure 2: Top left: top view of the single particle and the upper moving belt in $x-y$ coordinates (schematic and photograph); bottom right: oblique view of a schematic showing the fluid and the solid particle in $x-y-z$ coordinates.

Table 1: Stratified fluid properties.

\begin{tabular}{ccc}
\hline & $\rho\left(\mathrm{kg} / \mathrm{m}^{3}\right)$ & $v\left(\mathrm{~cm}^{2} / \mathrm{sec}\right)$ \\
\hline Upper layer fluid & 1110 & 146.2 \\
\hline Lower layer fluid & 1280 & 146.2 \\
\hline
\end{tabular}

In DPM, the influence of particle is represented as a source term in the Navier-Stokes equations. The continuous phase equations are solved in conjunction with the tracking of particles. For dilute systems, the effect of particles on the flow is negligible and the source term is set to zero. The continuous phase eqns are:

$$
\begin{gathered}
\frac{\partial \rho}{\partial t}+\frac{\partial}{\partial x_{j}}\left[\rho u_{j}\right]=0, \\
\frac{\partial}{\partial t}\left(\rho u_{i}\right)+\frac{\partial}{\partial x_{j}}\left[\rho u_{i} u_{j}+p \delta_{i j}-\tau_{i j}\right]=0, \quad i=1,2 .
\end{gathered}
$$


The motion of every individual particle with mass $m_{p}$ and velocity $u_{p}$ in the system is evaluated using Newton's second law:

$$
m_{p} \frac{d u_{p}}{d t}=F_{\text {drag }}+F_{\text {virtual mass }}+F_{\text {pressure }}+F_{\text {gravity }}+F_{\text {lift }} .
$$

The force terms on the right-hand side of eqn (3) are respectively drag, pressure, virtual mass, gravity, and lift.

\section{RESULTS AND DISCUSSION}

As mentioned earlier, the computational domain reflects the region in our experimental setup which is monitored and imaged by using a camera (top view). The primary phase velocity was found by the exact solution for the Couette flow domain. Some of the terms on the RHS of eqn (3) including the gravity, lift and virtual mass forces are negligible in this two-dimensional analysis. Eqn (3) can be rewritten as:

$$
\frac{d u_{p}}{d t}=C_{d r a g}\left(u_{f}-u_{p}\right)+C_{\text {virtual mass }}+C_{\text {pressure }} \text {. }
$$

Here $C_{\text {drag }}\left(u_{f}-u_{p}\right), C_{\text {pressure }}$ and $C_{\text {virtual mass }}$ represent the drag force, pressure gradient force and the virtual mass force respectively - all terms per unit mass. $C_{d r a g}$ is given as:

$$
C_{d r a g}=\frac{18 \mu}{\rho_{p} d_{p}^{2}} \frac{C_{d} R e}{24}
$$

where $u_{p}$ is the solid particle velocity, $C_{d}$ is the drag coefficient, $\rho_{p}$ and $d_{p}$ are the density and diameter of the spherical particle. The Reynolds number is calculated based on the relative velocity of the primary (fluid) and secondary phase (solid) as:

$$
R e=\frac{\rho d_{p}\left|u_{f}-u_{p}\right|}{\mu}
$$

where $\rho$ is the density of the primary phase.

The second term in eqn (4), $C_{\text {virtual mass }}$, can be written as:

$$
C_{\text {virtual mass }}=\frac{1}{2}\left(\frac{\rho}{\rho_{p}}\right) \frac{d}{d t}\left(u-u_{p}\right) .
$$

$C_{\text {virtual mass }}$ represents the force per unit mass required to accelerate the fluid around the particle. The pressure gradient force per unit mass $\left(C_{\text {pressure }}\right)$ is defined as:

$$
C_{\text {pressure }}=\left(\frac{\rho}{\rho_{p}}\right) u_{p} \frac{\partial u}{\partial x}
$$

The pressure gradient term can be neglected as the acceleration of the fluid is negligibly small with respect to the nature of this case. Stated differently, the change of fluid velocity in the $x$ direction is almost zero in this steady state Couette flow domain.

Note that there can be another external force term in eqn (4) to represent the lift force. However, this term can also be neglected due to force balance in the $x$ direction while the net force in the $y$ direction balances with gravity due to density differences.

The particle velocity and displacement are calculated by integrating the two-dimensional governing equation of motion. These simulations are performed with the second order accuracy. In all numerical studies, $d t<10^{-6} \mathrm{sec}$ and $d x<10^{-4} \mathrm{~m}$. 
In Fig. 3, the left photograph shows the top view of the particle suspended in the stratified flow in the experimental setup. The middle image is from the two-dimensional CFD of the discretized fluid domain with the solid particle which reflects the experimental particle location in the fluid. The right image shows the velocity contour plot of the fluid in the domain with the moving boundary condition at the bottom. The top boundary condition is zero velocity on the mid-axis due to the symmetry of the domain. The moving bottom boundary condition reflects the moving belt in the experimental setup. Since the whole domain in the experimental set up consists of two belts which move in opposite directions with the same velocity, just half of the domain is considered in the simulation study.

Fig. 4 compares the simulation results against the experimental data. The experiment is repeated at least 5 times for each Reynolds number and the mean values are presented in this figure. One of the key elements in both experimental and computational analysis is the moving belt speeds that control the exerted shear rate. The moving belt speeds provides the boundary condition for the analysis in the computational domain. A mean velocity of the belt is measured and is used as the boundary condition in the numerical simulations. Fig. 4 shows that the simulation results are in good agreement with the experimental data.

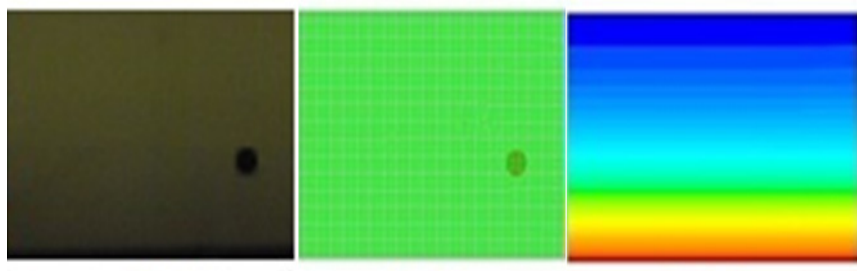

Figure 3: Left: particle located in the experimental setup (obtained from the experiment); Middle: particle located in the meshed primary phase; Right: fluid phase under the moving boundary condition from bottom.

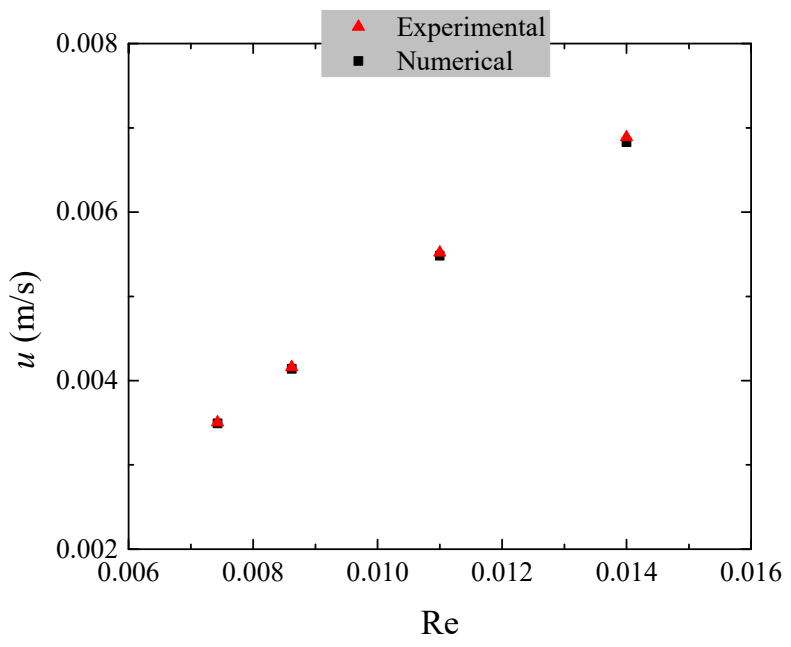

Figure 4: Computational and experimental particle velocity for values of the Reynolds number that were investigated. 


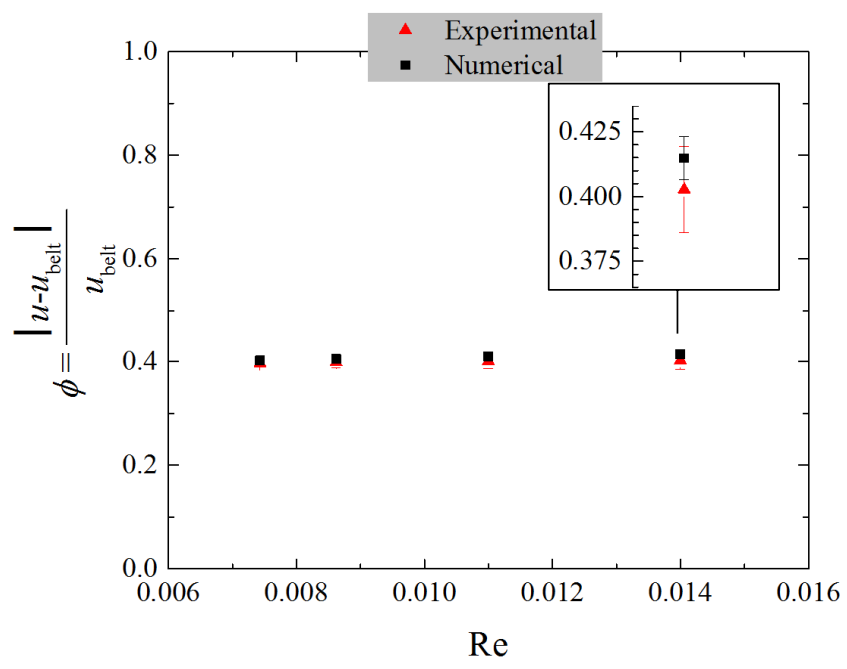

Figure 5: Comparison of the simulated relative dimensionless velocity with the experimental data for the range of Reynolds numbers under investigation.

During the experiments, we took measures to minimize the uncertainty in the positions of the moving boundaries. The compumotor drive to the pulleys moving the belts was implemented using a bicycle chain, and the belts and pulleys used a spring-loaded tightening arrangement, Nevertheless, occasional belt slippage did occur. Such slippage incidents are neglected in the evaluation of the mean values of the mean belt speed used in the simulation results shown in Fig. 4. To consider the effect of the sudden belt slippage during the experiments, the dimensionless relative velocity of the particle was defined as:

$$
\phi=\frac{u_{\text {belt }}-u_{p}}{u_{\text {belt }}} \text {. }
$$

Here $u_{\text {belt }}$ is the average belt velocity in the experimental setup. The uncertainties due to the belt slippage are considered in $\phi$ to provide a more realistic comparison between the computational results and the experimental data. The simulated dimensionless relative particle velocities, $\phi$, are compared with the experimental data in Fig. 5.

The value of $\phi$ converges to about 0.4 , and there is a good agreement between the simulation results and experimental data. Careful examination of Fig. 5 indicates that the simulated particle velocity shows a slight increase with the Reynolds number.

The uncertainties in both experimental and computational are also studied and quantified. The simulation results are sensitive to the initial location of the particle that was measured by the image processing that depends on the resolution of the images. This could cause an error of about $2 \%$ in the relative velocity which is presented in Fig. 5 on the simulation results. A variable uncertainty is observed in the experimental measurement due to the belt slippage. As the Reynolds number increases, the error bound of the experimental data for relative velocity increases. This can be attributed to several reasons including an increase in the belt slipping which contributes to $\phi$, increase in the contribution of rotation of particles, and their 
surface roughness. In addition, in the experimental study, the stratified primary phase consists of layers of fluid components with different densities, while in the simulation an average value of density of the fluid layers is used. Nevertheless, Fig. 5 shows that the simulation results are within the error bound where the error bound is mostly due to the uncertainties of the experimental data.

\section{CONCLUSIONS}

A combined computational-experimental study on the motion of a spherical particle in a low-Reynolds-number stratified shear flow was performed. An acceptable agreement was observed between the numerical results and the experimental data. Better agreement was observed at lower Reynolds numbers. At higher Reynolds numbers, the difference between the computational results and experimental data increases slightly which is more distinguished in $\phi$ since it has a direct contribution from the belt slippage. One of the main sources for the difference may be attributed to the stratified nature of the fluid in the experimental apparatus, while the average fluid density was used in the simulation. This issue, however, can be addressed in three-dimensional fluid flow analysis.

What are the implications of the findings? Usually comparison between experiment and numerics is conducted to validate the numerical modeling. However, this is not universally the case: for example, numerical modeling can also reveal deficiencies in experimental diagnostics [19]. In our case, the agreement between experiment and numerics both inspires confidence in the code and confirms that the uncertainties in the experimental setup are modest and the mechanisms producing them (e.g., belt slippage) do not need to be explicitly accounted for during modeling to result in good agreement. This suggests that the same experimental and numerical approach can be applied to future work: two-particle [12] and three-particle [13] interactions earlier studied in a cylindrical Couette cell, as well as interactions between non-spherical particles, both in Couette and Pouseuille flows. Two-particle interactions in periodic Couette flows in cylindrical geometry were shown to be irreversible [12], while three-particle [13], [14] and larger particle group [20] interactions can be interpreted as chaotic. Quantification of irreversibility/chaos in linear Couette/Pouseuille flow and elucidation of the underlying physics remain for future study.

\section{ACKNOWLEDGEMENT}

The experimental apparatus used in this study was originally developed with funding from the National Science Foundation grant 1335907.

\section{REFERENCES}

[1] Leal, L.G., Particle motions in a viscous fluid. Annual Review of Fluid Mechanics, 12, pp. 435-476, 1980.

[2] Ingber, M.S., Combined static and hydrodynamic interactions of two rough spheres in nonlinear shear flow. Journal of Rheology, 54(4), pp. 707-718, 2010.

[3] Ingber, M.S. \& Zinchenko, A., Semi-analytic solution of the motion of two spheres in arbitrary shear flow. International Journal of Multiphase Flow, 42, pp. 152-163, 2012.

[4] Ingber, M. \& Vorobieff, P., Particle interaction in oscillatory Stokes flow, WIT Transactions on Engineering Sciences, vol. 79, pp. 147-155, 2013.

[5] Fathi, N., Ingber, M. \& Vorobieff, P., Particle behavior in linear shear flow: An experimental and numerical study. Bulletin of the American Physical Society, 57, p. D8-005, 2012. 
[6] Fathi, N. \& Vorobieff, P., Spherical Particles in a Low Reynolds Number Flow: A V\&V Exercise. ASME Verification and Validation Symposium, V\&V2013-2281, p. 41, 2013.

[7] Mortazavi, S. \& Tryggvason, G., A numerical study of the motion of drops in Poiseuille flow. Part 1. Lateral migration of one drop. Journal of Fluid Mechanics, 411, pp. 325-350, 2000.

[8] Razi, M. \& Pourghasemi, M., Direct Numerical Simulation of deformable droplets motion with uncertain physical properties in macro and micro channels. Computer \& Fluids, 154(1), pp. 200-210, 2017.

[9] Chen, X., Xue, C., Zhang, L., Hu, G., Jiang, X. \& Sun, J., Inertial migration of deformable droplets in a microchannel. Physics of Fluids, 26(11), p. 112003, 2014.

[10] Nakayama, Y., Kim, K. \& Yamamoto, R., Simulating (electro) hydrodynamic effects in colloidal dispersions: Smoothed profile method. European Physical Journal E, 26(4), pp. 364-368, 2008.

[11] Nakayama, Y. \& Yamamoto, R., Simulation method to resolve hydrodynamic interactions in colloidal dispersions. Physical Review E, 71(3), p. 036707, 2005.

[12] Popova, M., Vorobieff, P., Ingber, M.S. \& Graham, A.L., Interaction of two particles in a shear flow. Physical Review E, 75(6), p. 066309, 2007.

[13] Jánosi, I.M., Tél, T., Wolf, D.E. \& Gallas, J.A., Chaotic particle dynamics in viscous flows: The three-particle Stokeslet problem. Physical Review E, 56(3), pp. 2858-2868, 1997.

[14] Popova, M., Vorobieff, P. \& Ingber, M., Analysis of two-and three-particle motion in a Couette cell. Computational Methods in Multiphase Flow IV, eds A.A. Mammoli \& C.A. Brebbia, WIT Press: Southampton, pp. 315-324, 2007.

[15] Tetlow, N., Graham, A.L., Ingber, M.S., Subia, S.R., Mondy, L.A. \& Altobelli, S.A., Particle migration in a Couette apparatus: Experiment and modeling. Journal of Rheology, 42(2), pp. 307-327, 1998.

[16] McNab, G.S. \& Meisen, A., Thermophoresis in liquids. Journal of Colloid and Interface Science, 44(2), pp. 339-346, 1973.

[17] Ingber, M. \& Vorobieff, P., Localized Scale Coupling and New Educational Paradigms in Multiscale Mathematics and Science. DOE Report DOE-UNM-25705, The University of New Mexico, 2014.

[18] ANSYS Fluent Theory Guide 18.0, Ansys Inc., 2017.

[19] Anderson, M. et al., An experimental and numerical study of shock interaction with a gas column seeded with droplets. Shock Waves, 25(2), pp. 107-125, 2015.

[20] Ingber, M.S., Mammoli, A.A., Vorobieff, P., McCollam, T. \& Graham, A.L., Experimental and numerical analysis of irreversibilities among particles suspended in a Couette device. Journal of Rheology, 50(2), pp. 99-114, 2006. 